\title{
Wavelength-tunable InAsP quantum dots in InP nanowires ${ }^{\circledR}$
}

Cite as: Appl. Phys. Lett. 115, 053101 (2019); https://doi.org/10.1063/1.5095675

Submitted: 12 March 2019 . Accepted: 26 June 2019. Published Online: 29 July 2019

Zhiqin Zhong, Xinlei Li ${ }^{(D)}$, Jiang Wu $\mathbb{D}^{-}$, Cheng Li, Ruo Bing Xie, Xiaoming Yuan, Xiaobin Niu (D), Wenhao Wang (D), Xiaorong Luo, Guojun Zhang, Zhiming M. Wang, Hark Hoe Tan (D), and Chennupati Jagadish

\section{COLLECTIONS}

EP This paper was selected as an Editor's Pick
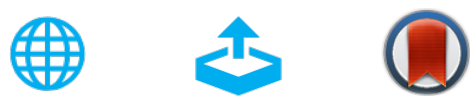

View Online

Export Citation

\section{ARTICLES YOU MAY BE INTERESTED IN}

Investigation of unintentional Fe incorporation in (010) $\beta-\mathrm{Ga}_{2} \mathrm{O}_{3}$ films grown by plasmaassisted molecular beam epitaxy

Applied Physics Letters 115, 052102 (2019); https://doi.org/10.1063/1.5096183

Mass measurement of graphene using quartz crystal microbalances

Applied Physics Letters 115, 053102 (2019); https://doi.org/10.1063/1.5111086

Space charge control of point defect spin states in AIN

Applied Physics Letters 115, 052101 (2019); https://doi.org/10.1063/1.5099916

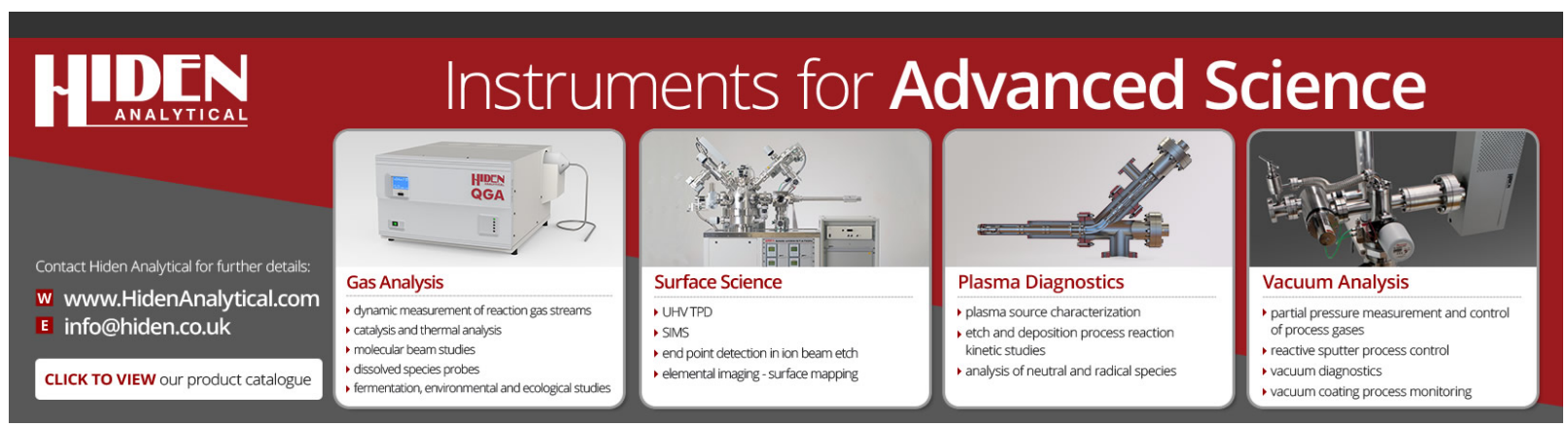




\title{
Wavelength-tunable InAsP quantum dots in InP nanowires
}

\author{
Cite as: Appl. Phys. Lett. 115, 053101 (2019); doi: 10.1063/1.5095675 \\ Submitted: 12 March 2019 - Accepted: 26 June 2019 . \\ Published Online: 29 July 2019
}

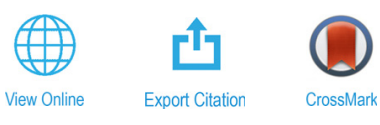

Zhiqin Zhong, ${ }^{1,2}$ Xinlei Li, ${ }^{3}$ (D) Jiang Wu, ${ }^{1,2, a)}$ (D) Cheng $\mathrm{Li}^{4}{ }^{4}$ Ruo Bing Xie, ${ }^{4}$ Xiaoming Yuan, ${ }^{5,6}$ Xiaobin Niu, ${ }^{1,2}$ Wenhao Wang, ${ }^{1,2}$ (D) Xiaorong Luo, Guojun Zhang, 'Zhiming M. Wang, ${ }^{1,2, a)}$ Hark Hoe Tan, $^{5, a)}$ (iD) and Chennupati Jagadish ${ }^{2,5}$

\begin{abstract}
AFFILIATIONS
${ }^{7}$ State Key Laboratory of Electronic Thin Films and Integrated Devices, University of Electronic Science and Technology of China, Chengdu 610054, China

${ }^{2}$ Institute of Fundamental and Frontier Science, University of Electronic Science and Technology of China, Chengdu 610054, China ${ }^{3}$ MOE Key Laboratory of Laser Life Science \& Institute of Laser Life Science, College of Biophotonics, South China Normal University, Guangzhou 510631, People's Republic of China

${ }^{4}$ Shanghai Institute of Applied Physics, Chinese Academy of Sciences, 2019 Jia Luo Road, Jiading District, Shanghai 201800, People's Republic of China

${ }^{5}$ Department of Electronic Materials Engineering, Research School of Physics and Engineering, The Australian National University, Canberra, ACT 2601, Australia

${ }^{6}$ School of Physics and Electronics, Hunan Key Laboratory for Supermicrostructure and Ultrafast Process, Central South University, 932 South Lushan Road, Changsha, Hunan 410083, People's Republic of China
\end{abstract}

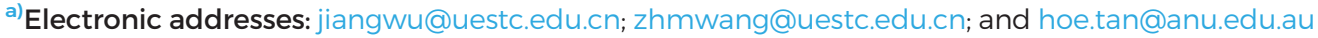

\begin{abstract}
There is considerable interest in quantum dots incorporated in nanowires for nanolasers and quantum emitters. In this letter, we demonstrate single InAsP quantum dots embedded in InP nanowires grown by metalorganic vapor-phase epitaxy. Despite the abrupt change of growth conditions at the interface, InAsP quantum dots can be grown in pure wurtzite InP nanowires. We develop a model and analyze the effects of the thickness of InAsP quantum dots and the composition of As on the formation of dislocations. Furthermore, the InAsP/InP quantum dot nanowires show bright photoluminescence up to room temperature without any surface passivation. The emission from the quantum dots could be well tuned by adjusting the dot size either vertically or laterally. The study demonstrates the potential of this material system for optoelectronic applications.
\end{abstract}

Published under license by AIP Publishing. https://doi.org/10.1063/1.5095675

Semiconductor nanowires (NWs) have many advantages, such as small footprint, large area-to-volume ratio, efficient strain relaxation in the formation of axial heterostructures, and the capability of efficient light harvesting by suppressing reflection over a broad range of wavelengths. ${ }^{1}$ NWs have been widely used in various devices, including light emitting diodes, lasers, solar cells, photodetectors, and transistors. $^{2-7}$ In particular, III-V semiconductors and metal oxide NWs have attracted tremendous research interest in the application of electronics $^{8-14}$ and optoelectronics ${ }^{15-18}$ due to their specific electronic and optoelectronic properties. Quantum dots (QDs) embedded in nanowire (NW) waveguides show great potential in lasers, solid-state single photon sources, and generating entangled photon pairs for quantum cryptography and quantum computation. ${ }^{19-25}$ Particularly, a single quantum dot positioned in a nanowire is considered as an ideal candidate for single photon emitters to achieve near-unity light-extraction efficiency. ${ }^{26}$ Also, it is reported that a photodetector made from $\mathrm{ZnO}$ quantum dot-decorated $\mathrm{Zn}_{2} \mathrm{SnO}_{4}$ nanowires demonstrates about 10 times higher photocurrent and responsibility compared with those based on pristine nanowires. ${ }^{14}$ So far, for nanowire quantum dots (NWQDs), there are mainly two methods to fabricate this kind of structure. One is the top-down approach by lithography followed by the dry etching process ${ }^{27}$ and the other one is the bottom-up approach. ${ }^{28-30}$ The drawback of top-down approaches is that quantum dots can possibly deviate from the center of the nanowire main axis, leading to a significant decrease in the efficiency of the single-photon source. $^{31}$ Additionally, light-extraction efficiencies in top-down 
approaches are limited by fabrication imperfections and etchinginduced defects, ${ }^{28}$ while the bottom-up approach can precisely locate the quantum dot in the center of the nanowire. ${ }^{29}$ The quantum-dot size and nanowire shape can also be controlled by the bottom-up approach. ${ }^{29}$ In addition, their small diameters enable the direct growth of III-V semiconductor nanowires on Si substrates without the formation of a high density of dislocations. ${ }^{32,33}$ Semiconductor NWs can be grown by the vapor-liquid-solid (VLS) method utilizing Au seed particles, ${ }^{34}$ self-catalysis, ${ }^{35}$ selective-area epitaxy (SAE), ${ }^{36}$ and Au-assisted SAE. ${ }^{22}$ By using the VLS method, a number of radial heterostructures have been reported and been applied for many applications. ${ }^{37,38}$ However, axial heterostructures are often less explored by the VLS method due to the vulnerability of the catalyst droplet to variation in growth conditions. Recently, group III-V semiconductor NWs containing insertions of a smaller bandgap, e.g., InAsP embedded into InP NWs, received much attention and were reported for the growth of axial nanowire quantum structures, e.g., quantum dots. ${ }^{22,34,39-41}$

For the nanowire quantum dot structure, the microstructure of the QD region and its proximity are very important to the excitonic emission. ${ }^{42}$ The structural quality of the QD plays a key role in producing indistinguishable photons, which is important for many quantum information applications. ${ }^{43}$ In GaSb and other Sb containing systems, it is shown that NW growth critically depends on the growth conditions, even for small variations of the local environment. ${ }^{44}$ Growth control of this structure is of fundamental interest and critical to enable high performance nanowire devices. In this letter, we tackle the challenges of growing high quality QDs in NWs with abrupt switching of growth conditions. InAsP/InP nanowires were synthesized using the Au-assisted vapor-liquid-solid method by metal organic vapor phase epitaxy (MOVPE). We report InAsP QDs imbedded in InP NWs. Bright emission was observed up to room temperature and with tunable emission wavelength.

The arrays of InAsP/InP nanowire heterostructures were grown in an AIXTRON 200/4 MOVPE reactor. The growth was carried out at a base pressure of 100 mbar, using ultrahigh purity $\mathrm{H}_{2}$ carrier gas with a total flow of $14.51 / \mathrm{min}$. Prior to growth, semi-insulating InP (111)B substrates were immersed in poly-L-lysine (PLL) solution for $60 \mathrm{~s}$. After being rinsed with de-ionized (DI) water, the substrates were blown dry with nitrogen gas. Then, a droplet of colloidal solution of Au particles was applied to the substrates. Au particles with diameters of 20,30 , or $40 \mathrm{~nm}$ were used. The substrates were then loaded into the reactor where InP NWs were first grown at $730^{\circ} \mathrm{C}$ with a V/III ratio of 80 . The flow rate for trimethylindium and phosphine was $6.07 \times 10^{-6} \mathrm{~mol} / \mathrm{min}$ and $4.91 \times 10^{-4} \mathrm{~mol} / \mathrm{min}$, respectively. After that, arsine $\left(\mathrm{AsH}_{3}\right)$ was added as the precursor for the growth of InAsP QD at the same growth temperature. For each size of Au particles, the QD growth duration was changed from 1 to $3 \mathrm{~s}$, to investigate the influence of both the microstructural and optical properties of the QDs. All other growth parameters were kept unchanged. After InAsP QD growth, arsine flow was switched off and the top InP segment was grown with the same condition as the bottom one. The InAsP/InP NWs were structurally characterized by field-emission scanning electron microscopy (FESEM) and high resolution transmission electron microscopy (TEM). The optical properties of single InAsP/InP NWs were assessed by room temperature microphotoluminescence ( $\mu$-PL) and temperature dependent photoluminescence using a confocal micro-Raman system (Horiba Jobin Yvon 64000) equipped with a $532 \mathrm{~nm}$ laser. The composition of QDs was measured using an energy dispersive X-ray (EDX) spectrometer equipped with a transmission electron microscope (TEM).

Figures $1(\mathrm{a})-1$ (c) display the FESEM images $\left(30^{\circ}\right.$ tilted $)$ of the nanowires grown with three different $\mathrm{Au}$ particle sizes where $3 \mathrm{~s}$ of $\mathrm{AsH}_{3}$ flow was used for the QD growth. The nanowires show a tapered needle shape with a wide base and a narrow tip. The tapered shape is attributed to surface diffusion of adatoms in regions between the nanowires which then deposited on the sidewalls of nanowires. ${ }^{45}$ Figure 1(d) exhibits the SEM image and the corresponding schematic illustration of a typical InP NW containing a single InAsP QD. The QD segment is marked by pink color. As shown in the magnified SEM image in Fig. 1(d), the base diameter is about $500 \mathrm{~nm}$, while the tip is as narrow as $50 \mathrm{~nm}$ in diameter.

Figure 2 shows the TEM image of a representative single InP $\mathrm{NW}$ with an embedded InAsP QD (1 s InAsP growth and $30 \mathrm{~nm} \mathrm{Au}$ particle) and the corresponding compositional mapping analysis using an energy dispersive X-ray (EDX) detector. The selected area diffraction pattern shown in Fig. 2(a) indicates a pure wurtzite (WZ) crystal structure. It should be noted that other nanowire quantum dot (NWQD) samples also show the WZ phase (e.g., supplementary material Fig. S1). As displayed in Fig. 2(b), a small dark area (marked by the green arrow) inside the InP NW is attributed to the formation of an InAsP QD, which is confirmed by EDX elemental mapping. The scanning area for the EDX mapping is $120 \mathrm{~nm} \times 120 \mathrm{~nm}$ as indicated by the dark blue dotted square in Fig. 2(b). Figures 2(c)-2(e) show the EDX mapping results for In, As, and P elements, respectively. While Fig. 2(c) shows a uniform In composition along the length of the NW, Fig. 2(e) shows a slightly reduced concentration of $\mathrm{P}$ in the region where the dark spot is observed in Fig. 2(b). Moreover, the As signal is detected in the same region as indicated in Fig. 2(d), thereby unambiguously confirming that an InAsP quantum dot was embedded in the InP NW.

The TEM images of the nine different NWQD samples grown with different QD growth times and with different nanoparticle sizes
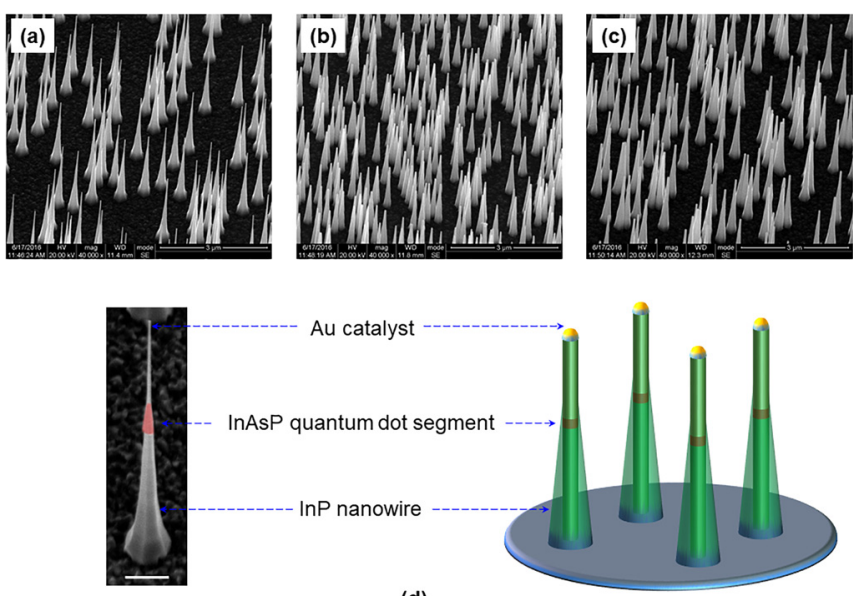

FIG. 1. FESEM images of InAsP/InP nanowires grown with a $3 \mathrm{~s}$ duration but different Au particle diameters: (a) $40 \mathrm{~nm}$, (b) $30 \mathrm{~nm}$, and (c) $20 \mathrm{~nm}$. (d) SEM image and the corresponding schematic illustration of the NW containing the InAsP quantum dot. The scale bar in (d) is $500 \mathrm{~nm}$. 


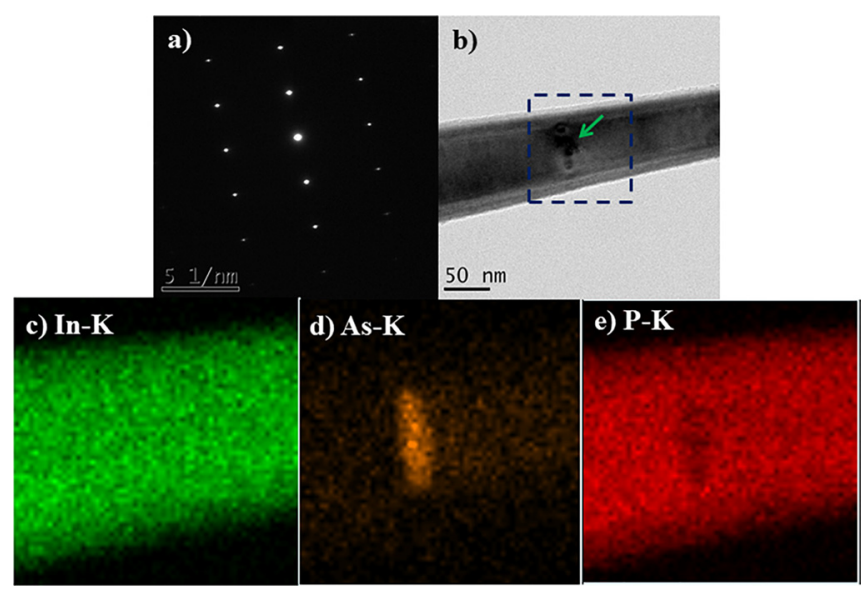

FIG. 2. (a) Selected area electron diffraction pattern of an InAsP/InP nanowire grown with a QD growth time of $1 \mathrm{~s}$, showing the WZ phase. (b) TEM image of the InAsP/InP NWQD. The dark blue dashed line shows the area where element mapping analysis was taken using an EDX detector. (c) to (e) EDX element mapping images of In, As, and $P$, respectively.

are shown in Fig. 3. All nanowires show a dark region, i.e., InAsP QD embedded in the InP nanowires. According to the image shown in Fig. 2(d), the dot is about $45 \mathrm{~nm}$ in diameter and $15 \mathrm{~nm}$ in height, which is much larger than a regular quantum dot. ${ }^{28}$ This conclusion is also supported by the TEM images as shown in Fig. 3. For example, based on the images of Figs. 3(a)-3(c), for the QDs grown for $1 \mathrm{~s}$, the diameter and the height of the dot range from $26 \mathrm{~nm}$ to $55 \mathrm{~nm}$ and

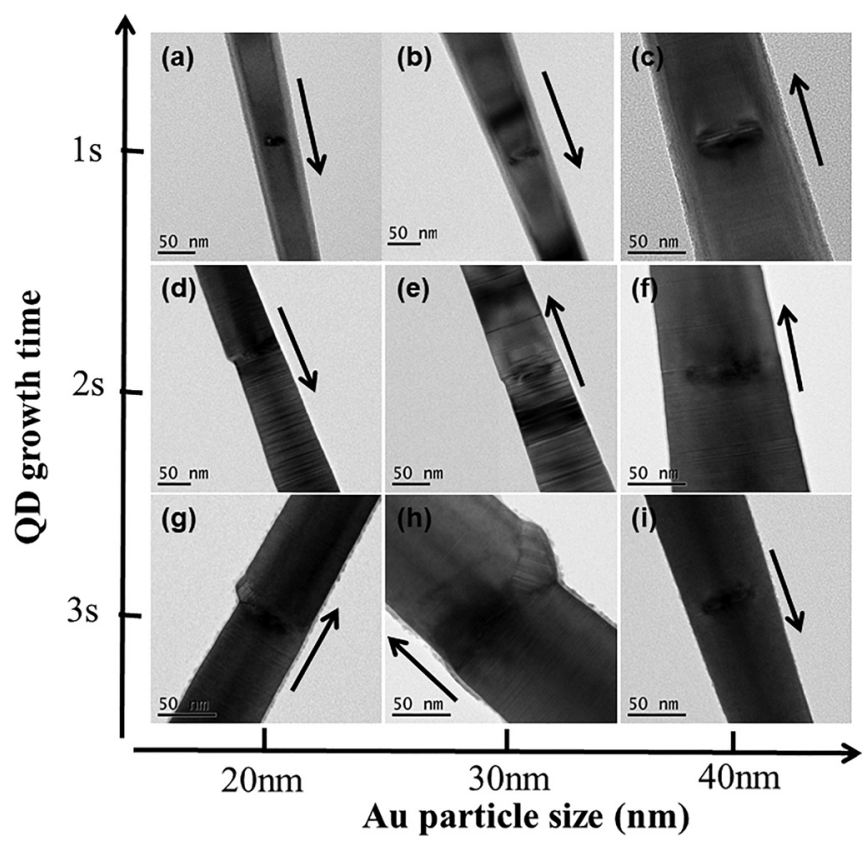

FIG. 3. TEM images of InAsP/InP NWs grown with different InAsP growth times and Au particle sizes. The scale bars are $50 \mathrm{~nm}$. The arrows indicate the NW growth direction. from $12 \mathrm{~nm}$ to $27 \mathrm{~nm}$, respectively, with the increasing $\mathrm{Au}$ nanoparticle size. For the QDs with a $40 \mathrm{~nm}$ Au particle, as shown in the 3rd column row of Fig. 3, the diameter and the height are in the range of $47 \mathrm{~nm}-60 \mathrm{~nm}$ and $20 \mathrm{~nm}-27 \mathrm{~nm}$, respectively. What's more, from Fig. 2(d), the As component shows the inhomogeneous distribution in the QD.

Generally, with the increasing Au catalyst size, the diameter of NWs increases. However, for the QDs grown for $3 \mathrm{~s}$, the evolution of the diameter of the QD region does not show a monotonic trend. This is due to the formation of lumps on the sidewall of nanowires where the QDs are located. These lumps can also be observed in the SEM images in Fig. 1 and the magnified SEM images (supplementary material Fig. S2). The formation of the lump on the NWQD sidewalls is attributed to the formation of dislocations during the QD growth, as shown by TEM results in Fig. S3. With the increasing QD growth time, the density of these defects increases. When arsine is introduced during the growth of the QD, supersaturation and/or energetics of the Au catalyst can be modified or InAsP is not lattice matched to InP, which then leads to the nucleation of these defects. ${ }^{46}$

To further understand the formation mechanism of dislocations or lattice distortion, either stacking faults or tilts, in the QD region with a long growth time $(\geq 2 \mathrm{~s})$, we performed a theoretical study. Supplying arsine for a long duration corresponds to a larger $\operatorname{InAs} \mathrm{P}_{1-\mathrm{x}} \mathrm{QD}$ and a high composition of As. Therefore, we analyze the effects of the thickness of InAs $\mathrm{P}_{1-\mathrm{x}} \mathrm{QD}$ and the composition of As on the formation of dislocations. The conclusion is that both the thicker thickness and the higher composition of As in $\operatorname{InAs}_{x} \mathrm{P}_{1-\mathrm{x}}$ are conducive to the formation of misfit dislocations (for details, see the supplementary material). The cause of the formation of misfit dislocations in the case of a large thickness and high composition of As is that the formation of dislocations can effectively release the strain stored in InAs $\mathrm{P}_{1-\mathrm{x}}$. As a result, dislocations can be observed from the samples grown with a longer QD growth duration. However, for a small thickness or low composition of As, though the formation of dislocations also releases the strain, the strain energy arising from dislocation pairs is larger than the release energy, which results in the higher total energy for $n=1$ than that for $n=0$.

Figures 4(a)-4(c) show the $300 \mathrm{~K}$ PL spectra of single NWQD from the set of samples. Almost all spectra show two distinct peaks. As an example, the blue curve in Fig. 4(a), corresponding to the PL spectrum of the NWQD grown with the $20 \mathrm{~nm}$ Au nanoparticle and $1 \mathrm{~s}$ of growth time, is fitted by the purple and green lines. The main peak centered at about $870 \mathrm{~nm}$ originates from the band-to-band transition in wurtzite dominated InP emission. ${ }^{47-49}$ The second and broader peak appearing at a longer wavelength may originate from the InAsP QD. This assumption is supported by the red-shift of this peak with the $\mathrm{AsH}_{3}$ supply duration. With the increasing QD growth time, the peak of this band shifts toward a longer wavelength, indicating the change of the quantum confinement effect. Compared to the emission peak of a typical single QD, it is clearly seen that the peak from QD is very broad. We think that there are two possible reasons for the broad linewidth. One possible reason is the large size of the QD, as mentioned in the TEM section. The large size of the QD leads to a large variation in the QD height along the growth direction. Another possibility of the broadening may be due to the inhomogeneous composition distribution in the large QD, which can also be inferred from the EDX measurements. For all samples with different catalyst sizes, 


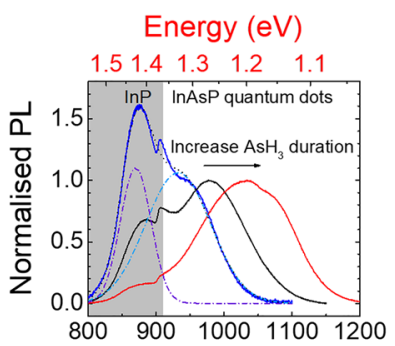

(a) Wavelength $(\mathrm{nm})$

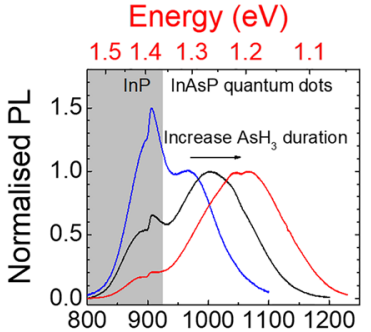

(c) Wavelength $(\mathrm{nm})$

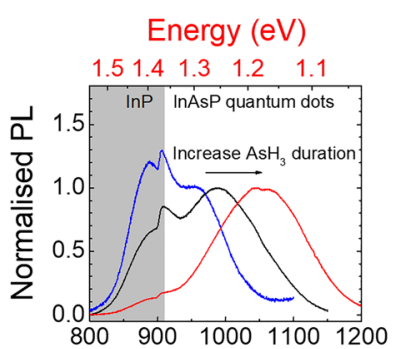

(b) Wavelength $(\mathrm{nm})$

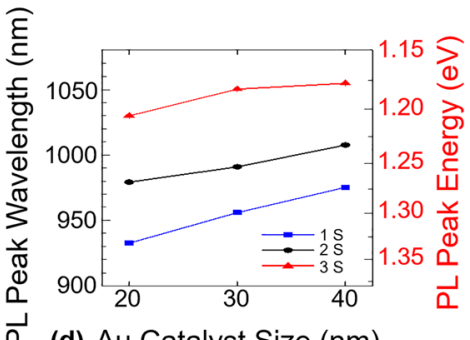

(d) Au Catalyst Size (nm)
FIG. 4. Room temperature $\mathrm{PL}$ spectra of $\ln A s P / \ln P$ NWs grown with different growth times and Au particle sizes. (a) $20 \mathrm{~nm}$; (b) $30 \mathrm{~nm}$; (c) $40 \mathrm{~nm}$. For each group of curves in (a)-(c), the blue ones are the PL spectra of NWQDs with a $1 \mathrm{~s}$ growth time for $\mathrm{AsH}_{3}$, the black one is $2 \mathrm{~s}$, and the red one is $3 \mathrm{~s}$. (d) The PL peak positions of the InAsP QD as a function of Au catalyst size grown for different growth times.

increasing the QD growth duration leads to a red-shift of the QD peak and a relative increase in intensity with respect to the InP peak. With the increasing growth time, the QD appears to have better confinement and hence shows a stronger PL signal. However, as shown in Figs. 3 and $\mathrm{S} 3$, the increase in the $\mathrm{AsH}_{3}$ deposition time has only a marginal effect on the height and size of the QD, for a fixed size of the $\mathrm{Au}$ catalysts. Therefore, it is reasonable to attribute the red-shift of the QD emission with the increasing $\mathrm{AsH}_{3}$ deposition time to a higher As concentration in the QD. This assumption is in agreement with the generation of misfit defects and tilts that are caused by the longer $\mathrm{AsH}_{3}$ deposition time $(\geq 2 \mathrm{~s})$. As a result, the emission from the QD can be well tuned by controlling the $\mathrm{AsH}_{3}$ supply time in addition to changing the size of Au catalyst, thereby allowing more flexibility, as shown in Fig. 4(d).

To gain further insight into optical properties of the NWQDs, temperature-dependent PL measurements were carried out for a single InAsP/InP NWQD grown with a $30 \mathrm{~nm}$ Au catalyst size and $3 \mathrm{~s}$ QD growth time, as shown in Fig. 5(a). At a low temperature of $7 \mathrm{~K}$, the emission width significantly reduced to 80 from $181 \mathrm{meV}$ measured at $300 \mathrm{~K}$. With increasing temperature, the emission broadens with a shoulder clearly emerging at a longer wavelength. The main PL peak follows the Varshni equation, indicating a single contribution from the NWQD (supplementary material Fig. S5). However, in Fig. 5(b), the FWHM shows a nonmonotonic dependence with temperature and a kink is clearly observed at around 150-200 K, suggesting thermal activation of carrier transfers from ground states to excited states within the NWQD or from different localized regions in the QD. This indicates that there are multiple optical transitions occurring in the NWQD.

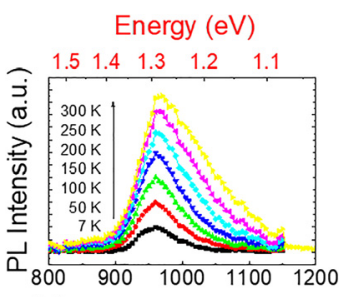

(a) Wavelength $(\mathrm{nm})$

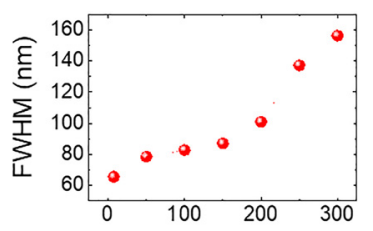

(b) Temperature $(\mathrm{K})$
FIG. 5. (a) Temperature dependence of $P L$ spectra of a single InAsP/InP QDNW grown with a $30 \mathrm{~nm}$ Au particle and a $3 \mathrm{~s} Q D$ growth time. The PL intensity is rescaled and evenly offset to guide the view of spectrum broadening. (b) Temperature dependence of the full width at half maximum (FWHM).

In conclusion, we have reported the growth of wavelengthtunable InAsP quantum dots within InP nanowires by the Au assisted VLS-MOVPE technique. The InP NWs embedded with an InAsP QD were grown by incorporating a low concentration of As in the Au catalyst. The low As concentration avoids a significant change of the heterostructure interface and the energetics of the catalyst that normally trigger tilt and formation of planar defects. PL measurements confirm QD emission in the infrared range, and the emission can be well tuned by changing the QD growth time and Au catalyst size. This work demonstrates the potential of using InAsP QDs within InP NWs for optoelectronic applications.

See the supplementary material for further details of experimental methods and measurements.

The authors are grateful to P. Caroff-Gaonac'h for technical growth of InAsP/InP NWQDs. The authors acknowledge the financial support from the Australian Research Council (ARC), National Natural Science Foundation of China (Grant No. 11305029), Natural Science Foundation of Guangdong Province (Grants Nos. 2017A030313389 and 2018A030313125), Fundamental Research Funds for the Central Universities (Grant No. ZYGX2016J054), National Basic Research Program (973) of China through Grant No. 2015CB358600, and Australian National Fabrication Facility (ANFF) ACT node for facility support.

\section{REFERENCES}

${ }^{7}$ Z. Arefinia and A. Asgari, J. Renewable Sustainable Energy 6(4), 043132 (2014).

${ }^{2}$ B.-H. Lee, J. Hur, M.-H. Kang, T. Bang, D.-C. Ahn, D. Lee, K.-H. Kim, and Y.-K. Choi, Nano Lett. 16(3), 1840 (2016).

${ }^{3}$ Y. Peng, M. Que, H. E. Lee, R. Bao, X. Wang, J. Lu, Z. Yuan, X. Li, J. Tao, J. Sun, J. Zhai, K. J. Lee, and C. Pan, Nano Energy 58, 633 (2019).

${ }^{4}$ Z. Zhong, Z. Li, Q. Gao, Z. Li, K. Peng, L. Li, S. Mokkapati, K. Vora, J. Wu, G. Zhang, Z. Wang, L. Fu, H. H. Tan, and C. Jagadish, Nano Energy 28, 106 (2016).

${ }^{5}$ X. Dai, S. Zhang, Z. Wang, G. Adamo, H. Liu, Y. Huang, C. Couteau, and C. Soci, Nano Lett. 14(5), 2688 (2014).

${ }^{6}$ Y. Zhang, D. Saxena, M. Aagesen, and H. Liu, Nanotechnology 30(19), 192002 (2019).

${ }^{7}$ M. D. Brubaker, K. L. Genter, A. Roshko, P. T. Blanchard, B. T. Spann, T. E. Harvey, and K. A. Bertness, Nanotechnology 30(23), 234001 (2019).

${ }^{8}$ Z. Zhou, C. Lan, R. Wei, and J. C. Ho, J. Mater. Chem. C 7(2), 202 (2019).

${ }^{9}$ L. Song, L. Luo, X. Li, D. Liu, N. Han, L. Liu, Y. Qin, J. C. Ho, and F. Wang, Adv. Electron. Mater. 5(3), 1800707 (2019). 
${ }^{10}$ E. Memisevic, M. Hellenbrand, E. Lind, A. R. Persson, S. Sant, A. Schenk, J. Svensson, R. Wallenberg, and L. E. Wernersson, Nano Lett. 17(7), 4373 (2017).

${ }^{11}$ D. Cutaia, K. E. Moselund, H. Schmid, M. Borg, A. Olziersky, and H. Riel, in IEEE Symposium on VLSI Technology (2016), p. 1.

${ }^{12}$ A. Jonsson, J. Svensson, and L.-E. Wernersson, IEEE Electron Device Lett. 39(7), 935 (2018).

${ }^{13}$ J. Svensson, A. W. Dey, D. Jacobsson, and L. E. Wernersson, Nano Lett. 15(12), 7898 (2015)

${ }^{14}$ L. Li, L. Gu, Z. Lou, Z. Fan, and G. Shen, ACS Nano 11(4), 4067 (2017).

${ }^{15}$ W. Ouyang, F. Teng, J.-H. He, and X. Fang, Adv. Funct. Mater. 29(9), 1807672 (2019).

${ }^{16}$ W. J. Lee, P. Senanayake, A. C. Farrell, A. Lin, C. H. Hung, and D. L. Huffaker, Nano Lett. 16(1), 199 (2016)

${ }^{17}$ M. D. Thompson, A. Alhodaib, A. P. Craig, A. Robson, A. Aziz, A. Krier, J. Svensson, L. E. Wernersson, A. M. Sanchez, and A. R. Marshall, Nano Lett. 16(1), 182 (2016).

${ }^{18} \mathrm{Z}$. Li, X. Yuan, L. Fu, K. Peng, F. Wang, X. Fu, P. Caroff, T. P. White, H. Hoe Tan, and C. Jagadish, Nanotechnology 26(44), 445202 (2015).

${ }^{19}$ J. Tatebayashi, S. Kako, J. Ho, Y. Ota, S. Iwamoto, and Y. Arakawa, Nat. Photonics 9(8), 501 (2015).

${ }^{20}$ J. Ho, J. Tatebayashi, S. Sergent, C. F. Fong, Y. Ota, S. Iwamoto, and Y. Arakawa, Nano Lett. 16(4), 2845 (2016).

${ }^{21}$ Y. Hu, H. O. Churchill, D. J. Reilly, J. Xiang, C. M. Lieber, and C. M. Marcus, Nat. Nanotechnol. 2(10), 622 (2007).

${ }^{22}$ D. Dalacu, K. Mnaymneh, J. Lapointe, X. Wu, P. J. Poole, G. Bulgarini, V. Zwiller, and M. E. Reimer, Nano Lett. 12(11), 5919 (2012).

${ }^{23}$ M. A. Versteegh, M. E. Reimer, K. D. Jöns, D. Dalacu, P. J. Poole, A. Gulinatti, A. Giudice, and V. Zwiller, Nat. Commun. 5, 5298 (2014).

${ }^{24}$ M. B.Bavinck, K. D. Jöns, M. Zieliński, G. Patriarche, J.-C. Harmand, N. Akopian, and V. Zwiller, Nano Lett. 16(2), 1081 (2016).

${ }^{25}$ A. W. Elshaari, I. E. Zadeh, A. Fognini, M. E. Reimer, D. Dalacu, P. J. Poole, V. Zwiller, and K. D. Jöns, Nat. Commun. 8(1), 379 (2017).

${ }^{26}$ I. Friedler, C. Sauvan, J. P. Hugonin, P. Lalanne, J. Claudon, and J. M. Gerard, Opt. Express 17(4), 2095 (2009).

${ }^{27}$ J. Claudon, J. Bleuse, N. S. Malik, M. Bazin, P. Jaffrennou, N. Gregersen, C. Sauvan, P. Lalanne, and J. M. Gerard, Nat. Photonics 4(3), 174 (2010).

${ }^{28}$ M. E. Reimer, G. Bulgarini, N. Akopian, M. Hocevar, M. B. Bavinck, M. A. Verheijen, E. Bakkers, L. P. Kouwenhoven, and V. Zwiller, Nat. Commun. 3, 737 (2012).

${ }^{29}$ M. E. Reimer, G. Bulgarini, E. Bakkers, D. Dalacu, P. J. Poole, and V. Zwiller, in Physics and Simulation of Optoelectronic Devices XXI, edited by B. Witzigmann, M. Osinski, F. Henneberger et al. (International Society for Optics and Photonics, SPIE, 2013), Vol. 8619.
${ }^{30}$ J. Wu, A. Ramsay, A. Sanchez, Y. Y. Zhang, D. Kim, F. Brossard, X. Hu, M Benamara, M. E. Ware, Y. I. Mazur, G. J. Salamo, M. Aagesen, Z. M. Wang, and H. Y. Liu, Nano Lett. 16(1), 504 (2016).

${ }^{31}$ J. Bleuse, J. Claudon, M. Creasey, N. S. Malik, J. M. Gerard, I. Maksymov, J. P. Hugonin, and P. Lalanne, Phys. Rev. Lett. 106(10), 103601 (2011).

${ }^{32}$ C. M. Haapamaki, J. Baugh, and R. R. LaPierre, J. Appl. Phys. 112(12), 124305 (2012).

${ }^{33}$ M. N. Mankin, R. W. Day, R. Gao, Y.-S. No, S.-K. Kim, A. A. McClelland, D. C. Bell, H.-G. Park, and C. M. Lieber, Nano Lett. 15(7), 4776 (2015).

${ }^{34}$ K. Tateno, G. Zhang, H. Gotoh, and T. Sogawa, Nano Lett. 12(6), 2888 (2012).

${ }^{35}$ J. Wu, Y. B. Li, J. Kubota, K. Domen, M. Aagesen, T. Ward, A. Sanchez, R. Beanland, Y. Y. Zhang, M. C. Tang, S. Hatch, A. Seeds, and H. Y. Liu, Nano Lett. 14(4), 2013 (2014).

${ }^{36}$ S. N. Dorenbos, H. Sasakura, M. P. van Kouwen, N. Akopian, S. Adachi, N. Namekata, M. Jo, J. Motohisa, Y. Kobayashi, K. Tomioka, T. Fukui, S. Inoue, H. Kumano, C. M. Natarajan, R. H. Hadfield, T. Zijlstra, T. M. Klapwijk, V. Zwiller, and I. Suemune, Appl. Phys. Lett. 97(17), 171106 (2010).

${ }^{37}$ F. Qian, Y. Li, S. Gradečak, H.-G. Park, Y. Dong, Y. Ding, Z. L. Wang, and C. M. Lieber, Nat. Mater. 7(9), 701 (2008).

${ }^{38}$ B. Mayer, D. Rudolph, G. Abstreiter, G. Bracher, G. Koblmüller, J. J. Finley, J. Schnell, J. Winnerl, J. Treu, and K. Müller, Nat. Commun. 4, 2931 (2013).

${ }^{39}$ D. Dalacu, K. Mnaymneh, X. Wu, J. Lapointe, G. C. Aers, P. J. Poole, and R. L. Williams, Appl. Phys. Lett. 98(25), 251101 (2011).

${ }^{40}$ J.-C. Harmand, F. Jabeen, L. Liu, G. Patriarche, K. Gauthron, P. Senellart, D. Elvira, and A. Beveratos, J. Cryst. Growth 378, 519 (2013).

${ }^{41}$ K. A. Dick, J. Bolinsson, B. M. Borg, and J. Johansson, Nano Lett. 12(6), 3200 (2012).

${ }^{42}$ M. Holmes, S. Kako, K. Choi, M. Arita, and Y. Arakawa, Phys. Rev. B 92(11), 115447 (2015).

${ }^{43}$ E. Knill, R. Laflamme, and G. J. Milburn, Nature 409(6816), 46 (2001).

${ }^{44}$ B. M.Borg and L.-E. Wernersson, Nanotechnology 24(20), 202001 (2013).

${ }^{45}$ J. H. Kang, Q. Gao, H. J. Joyce, H. H. Tan, C. Jagadish, Y. Kim, D. Y. Choi, Y. Guo, H. Xu, J. Zou, M. A. Fickenscher, L. M. Smith, H. E. Jackson, and J. M. Yarrison-Rice, Nanotechnology 21(3), 035604 (2010).

${ }^{46}$ S. A. Dayeh, J. Wang, N. Li, J. Y. Huang, A. V. Gin, and S. T. Picraux, Nano Lett. 11(10), 4200 (2011).

${ }^{47}$ L. V. Titova, T. B. Hoang, J. M. Yarrison-Rice, H. E. Jackson, Y. Kim, H. J. Joyce, Q. Gao, H. H. Tan, C. Jagadish, X. Zhang, J. Zou, and L. M. Smith, Nano Lett. 7(11), 3383 (2007).

${ }^{48}$ A. Mishra, L. V. Titova, T. B. Hoang, H. E. Jackson, L. M. Smith, J. M. Yarrison-Rice, Y. Kim, H. J. Joyce, Q. Gao, H. H. Tan, and C. Jagadish, Appl. Phys. Lett. 91(26), 263104 (2007).

${ }^{49}$ S. Paiman, Q. Gao, H. H. Tan, C. Jagadish, X. Zhang, and J. Zou, J. Cryst. Growth 383(Suppl. C), 100 (2013). 